\title{
To Professor Hvedri Inassaridze's 80th birthday
}

\author{
George Janelidze
}

Published online: 2 October 2013

(C) Tbilisi Centre for Mathematical Sciences 2013

This letter is about my former supervisor, who will see it, and I hope will forgive me for all its imperfections. It also says a few words about a remarkable event in the history of the Georgian science-the creation of the first and so far unique successful and internationally recognized Georgian school of abstract algebra. This creation was based on Inassaridze's mathematical talent, originality of thoughts, and mathematical achievements - especially in homological and homotopical algebra, algebraic topology, and algebraic $K$-theory. I only indicate some of these achievements, and the best way to learn about them is to read his papers and books of course; they are all listed at the end of the letter.

Hvedri Inassaridze was born in Paris on 6 December, 1932. As his webpage http:// www.rmi.ge/ hvedri/ says (but I am slightly changing the wording), he studied at Lycée Claude Bernard, Paris, until 1947, and then completed his school education in Tbilisi in 1950. In the same year he became a student of Tbilisi State University, but one year later his family was sent for the so-called permanent exile to Kazakhstan. By the way, I was also there for a similar reason, but we could not have known each other since Inassaridze family was rehabilitated in 1954, when I was two years old, not to mention that Kazakhstan is very large ...

Hvedri continued his study at Tbilisi State University, and graduated in 1958. A $\mathrm{PhD}$ ("Candidate of Science") degree in those years, and especially if your supervisor was George Chogoshvili, would mean that you are an established mathematician with several substantial publications. However, Hvedri's PhD dissertation was exceptionally good even at that level—so good that it was transformed into DSc dissertation. This involved a complicated bureaucratic procedure, and as a result, he became a Candidate of Science in 1966 and a Doctor of Science in 1970. For the readers not familiar with the Eastern European academic system, I should add: the difference between

\footnotetext{
G. Janelidze $(\varangle)$

University of Cape Town, Cape Town, South Africa

e-mail: George.Janelidze@uct.ac.za
} 
$\mathrm{PhD}$ and DSc was supposed to be enormous, e.g. about 3 publications versus 30, and often more than 20 years of work of an active researcher.

Let us look at Inassaridze's first 14 papers, which were published before 1970. Are they written by a universal algebraist $[1,4],{ }^{1}$ by a point-set topologist $\left([8],{ }^{2}[9]\right.$, and [11]) or by an expert in abstract homological algebra $[2,3,5-7,10,12,13]$ ? These his first papers are certainly already deep enough to ask this question, and the answer is that they were all written by the same growing distinguished mathematician whose main research topic in those years eventually turned out to be: the study of his very general notion of a satellite of a functor with respect to what he called a presheaf of categories with a trace, and of various examples of that notion. Inassaridze's satellites are completely general, that is, no exactness properties are used, and his presheaves of categories are essentially the same as contravariant pseudo-functors into CAT (=indexed categories), although he never required any coherence conditions. His ideas came up a few years later than those of Yoneda (satellites via regular spans) and of Grothendieck (Grotendieck fibrations, Grothendieck construction), but absolutely independently, and later inspired new ones, which he beautifully used in non-abelian homological algebra and algebraic $K$-theory.

Inassaridze begins his wonderful 'pro-seminar' with undergraduate students in 1969/70, where each student soon gets a topic of independent study and research, and he/she is supposed to report about the ideas and results he/she studied and the ideas and results he/she discovered. The topics were very diverse and often unrelated to Inassaridze's own research; but we (I was one of them) considered this as the best form of academic freedom, and we knew that Inassaridze's supervisor gave similar freedom to his students, and now we are trying the same with our students. Inassaridze's pedagogical success was unbelievable: in merely 4-5 years the word "algebraist", as used in either Razmadze Institute of Mathematics of the Georgian Academy of Science or in Mechanics-Mathematics Department of Tbilisi State University, has become almost a synonym of "either Hvedri Inassaridze, or his student". I would like to repeat (but in English) the last sentence of the letter I have sent to Razmadze Institute for the celebration of Inassaridze's 80th birthday: "I would list all your students and speak about their mathematical achievements with admiration, but let me just say again, that we are all so grateful to you!".

From early 70s Inassaridze's research is rather focused on non-abelian homological and homotopical algebra, on its relation to $K$-theory-where he is one of the pioneers and the author of a number of fundamental results-and on $K$-theory itself. In parallel to this, and also even earlier, he makes a significant contribution to (co)homology theories in algebraic topology (see [14-16] and joint work with Mdzinarishvili [24]); more recently he obtained an axiomatic characterization of Steenrod homology theory of compact spaces [34]. However, the above-mentioned homological algebra/ $K$-theory theme remains Inassaridze's most important topic of research from then to these days.

\footnotetext{
1 More precisely, [1] and [4] should be attributed to semigroup theory and to generalized group theory, respectively; semigroups also appear in Inassaridze's other papers in sixties.

2 Which introduces $n$-fold extensions and growths of completely regular spaces, extending, in a natural way, the class of locally compact spaces, and closely related to dimension theory of topological spaces as developed in works of L. Zambakhidze and I. Tsereteli.
} 
About 30 of Inassaridze's publications are devoted to it, and, not trying to describe their wide range of new ideas and results, let me only recall/mention (the sentences in italic are copied from either papers or communication with Inassaridze):

- Definition of non-abelian derived functors of group valued functors with respect to projective classes and applications to K-theory [22].

- New approach to equivariant theory of homology and cohomology of groups with application to algebraic $K$-theory [52].

- Realization of K-theory of normed algebras showing that Quillen's construction for normed algebras gives us both algebraic and topological K-theories [43].

- Introduction (with T. Kandelaki) [56] of smooth K-theory of locally convex algebras to confirm the smooth Karoubi Conjecture on the isomorphism of algebraic and topological K-functors for quasi stable Frechet algebras with properly uniformly bounded approximate unit.

- His interest in K-theory makes Inassaridze's work "less abstract-categorical” (I was disappointed, but a student is not in the position to tell his supervisor what to do...). However, categories had appeared again in his work in the form of crossed modules (starting from [36]) and categorical groups (see [44] and [49]).

- As we can see from the list of his papers, Inassaridze collaborated (so far) with D. Arlettaz, A. M. Cegarra, D. Conduché, A. Del Río, A. R. Garzón, N. Inassaridze (his son), T. Kandelaki, E. Khmaladze, L. Mdzinarishvili, and R. Meyer. The Georgian collaborators were also his former students (except Mdzinarishvili). Several other former students of Inassaridze greatly developed some of his ideas and added new ones (and also worked on many other things). This includes (ordered chronologically): A. Patchkoria (homological algebra of monoids and semimodules), T. Pirashvili (non-abelian derived functors and $K$-theory), D. Pataraia ( $K$-theory). My own small contribution was related to his early work on satellites. But again, Inassaridze equally appreciated and supported good work of all his students, even if it was unrelated to his own work.

- Inassaridze is the author of three books (so far): [20], [35], and [38]. [20] represents six topics/directions of the author's research in homological and homotopical algebra, and its purpose is simply to put together the author's results in those directions; [38] repeats and revises some material on [20], which is helpful since the book [20] is almost impossible to get, and adds a lot more, including many results of the author's students; [35] is rather a textbook, a very advanced one, in (many aspects of) $K$-theory, first of all algebraic, but also topological. Although the main purpose of [35] is to present the author's results related to the two ideas above (see the first two bullet-points), it is also particularly useful to study various known higher $K$-theories; apart from that and other related things it has a chapter devoted to J. Gubeladze's and R. G. Swan's proofs of D. F. Anderson's generalization of J.-P. Serre's conjecture (on "projective=free" for modules over polynomial rings).

To many of us, Georgian algebraists and topologists, the Georgian modern mathematics begins with George Chogoshvili, but we never think of Hvedri Inassaridze as simply one of Chogoshvili's followers. Inassaridze had made a great next step towards abstract algebra, made a good contribution to it, and created his own internationally recognized school, as already mentioned. He also was elected, as a Corresponding 
Member in 1983 and then as a Full Member in 2001, to the Georgian Academy of Sciences (without ever being a Communist Party Member, just as Chogoshvili and Chogoshvili's another former student, Nodar Berikashvili). In 1990-1991 Inassaridze was a Member of Georgian Parlament, but surely, as a greatly respected scientist rather than a politically active person. In 1990-1997 he was the President of the Georgian National Mathematical Committee. For many years he was (and presently is) Head of the Algebra Department of A. Razmadze Mathematical Institute, and Professor of Tbilisi State University. Inassaridze is the founder of three international mathematical journals: Homology, Homotopy and Applications, where he was Editor-in-Chief in 1995-2008, and Journal of Homotopy and Related Structures and Tbilisi Mathematical Journal, where he still is Editor-in-Chief; he also is a member of the Editorial Board of the Georgian Mathematical Journal.

Some of Inassaridze's former students, who defended their $\mathrm{PhD}$ dissertations under his supervision, were mentioned above. The full list is: T. Datuashvili, J. Gubeladze, Z. Janelidze, T. Kandelaki, R. Katamadze, E. Khmaladze, A. Landia, Nguyen Xuan Tuyen, A. Patchkoria, B. Pachuashvili, T. Pirashvili, G. Rakviashvili, E. Rtveliashvili, M. Uridia, and myself.

\section{List of Publications of Hvedri Inassaridze}

1. On simple semigroups. Mat. Sb. (N.S.) 57(99), 225-232 (1962)

2. On the theory of extensions in categories. Bull. Georgian Acad. Sci. 30, 537-544 (1963)

3. Extensions of semigroups. Bull. Georgian Acad. Sci. 33, 263-269 (1964)

4. On the theory of generalised heaps. Proc. Tbilisi State Univ., Ser. Mech.-Math. Sci. 102, 207-210 (1964)

5. Universal functors. Bull. Georgian Acad. Sci. 38, 513-520 (1965)

6. Extensions of regular semigroups. Bull. Georgian Acad. Sci. 39, 3-10 (1965)

7. Derived functors. Bull. Georgian Acad. Sci. 39, 527-533 (1965)

8. On extensions and growths of finite order for completely regular spaces. Dokl. Akad. Nauk SSSR 166, 1043-1045 (1966)

9. A generalization of perfect mappings. Dokl. Akad. Nauk SSSR 168, 266-268 (1966)

10. Extensions of semigroups with a zero-element. Bull. Georgian Acad. Sci. 41, 513-520 (1966)

11. A generalization of closed mappings and the absolutes of the $n$th order. Bull. Georgian Acad. Sci. 42, 513-519 (1966)

12. Extensions of commutative inverse semigroups. Bull. Georgian Acad. Sci. 46, 11-18 (1967)

13. Universal functors in general categories. Bull. Georgian Acad. Sci. 50, 541-546 (1968)

14. Alexander-Kolmogorov cohomology with values in commutative inverse semigroups. Bull. Georgian Acad. Sci. 54, 269-272 (1969)

15. Exact homology and linking for Steenrod duality. Dokl. Akad. Nauk SSSR 206, 21-24 (1972)

16. Exact homology. Proc. A. Razmadze Math. Institute 41, 128-142 (1972), 165 (1973)

17. The exact homology and Tate cohomology for locally compact zero-dimensional groups. Bull. Georgian Acad. Sci. 74, 29-32 (1974)

18. Homotopy of pseudosimplicial groups and nonabelian derived functors. Bull. Georgian Acad. Sci. 76, 533-536 (1974)

19. A generalization of the Milnor sequence for inverse limits. Bull. Georgian Acad. Sci. 79, 17-20 (1975)

20. Certain questions of homological and homotopical algebra and their applications. In: Proceeding of A. Razmadze Math. Institute 48 (1975) "Mecniereba", Tbilisi, p. 141 (1975)

21. Algebraic $K$-functors. Bull. Georgian Acad. Sci. 77, 17-20 (1975)

22. Homotopy of pseudosimplicial groups, nonabelian derived functors, and algebraic $K$-theory. Mat. Sb. (N.S.) 98(140), 3(11), 339-362, 495 (1975)

23. The stabilization of the Karoubi-Villamayor algebraic $K$-functors. Bull. Georgian Acad. Sci. 85, 29-32 (1977) 
24. (With L. Mdzinarishvili) The interconnection between continuity and exactness in homology theory. Bull. Georgian Acad. Sci. 99, 317-320 (1980)

25. Stabilization of algebraic $K$-functors. Bull. Georgian Acad. Sci. 103, 21-24 (1981)

26. On the natural mappings between algebraic and topological $K$-theories. Bull. Georgian Acad. Sci. 103, 277-280 (1981)

27. The functor $K_{3}$ of Swan and Gersten. Bull. Georgian Acad. Sci. 111, 29-31 (1983)

28. $K$-theory of special normed algebras. Uspekhi Mat. Nauk 40, 4(244), 169-170 (1985)

29. Swan's $K$-theory for Banach algebras over a unitary Banach ring. Bull. Georgian Acad. Sci. 118, 469-472 (1985)

30. Characterization of exact bifunctor homology theories. Bull. Georgian Acad. Sci. 121, 241-243 (1986)

31. On the homotopization of Karoubi-Villamayor algebraic $K$-theory. Bull. Georgian Acad. Sci. 125, 17-19 (1987)

32. $K$-theory of special normed rings. Bull. Georgian Acad. Sci. 134, 57-60 (1989)

33. $K$-theory of special normed rings. $K$-theory and homological algebra (Tbilisi, 1987-88), pp. 95-156, Lecture Notes in Math. 1437, Springer, Berlin (1990)

34. On the Steenrod homology theory of compact spaces. Michigan Math. J. 38(3), 323-338 (1991)

35. Algebraic $K$-theory. Mathematics and its Applications, 311. Kluwer Academic Publishers Group, Dordrecht, p. viii+438. ISBN: 0-7923-3185-0 (1995)

36. Non-abelian cohomology of groups. Georgian Math. J. 4(4), 313-332 (1997)

37. Non-abelian cohomology with coefficients in crossed bimodules. Georgian Math. J. 4(6), 509-522 (1997)

38. Non-abelian homological algebra and its applications. In: Mathematics and its Applications, 421, Kluwer Academic Publishers, Dordrecht, p. iv+265. ISBN: 0-7923-4718-8 (1997)

39. Universal property of Kasparov bivariant $K$-theory. Bull. Georgian Acad. Sci. 156(2), 185-189 (1997)

40. (With N. Inassaridze) New descriptions of the non-abelian homology of groups. Bull. Georgian Acad. Sci. 157(2), 196-199 (1998)

41. (With N. Inassaridze) The second and the third non-abelian homology of groups. Bull. Georgian Acad. Sci. 158(3), 376-378 (1998/9)

42. (With N. Inassaridze) Non-abelian homology of groups. $K$-Theory 18(1), 1-17 (1999)

43. Algebraic $K$-theory of normed algebras. $K$-Theory 21(1), 25-56 (2000)

44. (With A. R. Garzón) Semidirect products of categorical groups. Obstruction theory. Homology Homotopy Appl. 3(1), 111-138 (2001)

45. (With T. Kandelaki) $K$-theory of stable generalized operator algebras. $K$-Theory 27(2), 103-110 (2002)

46. Higher non-abelian cohomology of groups. Glasgow Math. J. 44(3), 497-520 (2002)

47. (With A. M. Cegarra) Homology of groups with operators. Int. Math. J. 5(1), 29-48 (2004)

48. (With D. Conduché and N. Inassaridze) Mod $q$ cohomology and Tate-Vogel cohomology of groups, J. Pure Appl. Algebra 189(1-3), 61-87 (2004)

49. (With A. R. Garzón and A. del Río) Derivations of categorical groups. Theory Appl. Categ. 13(5), 86-105 (2004)

50. More about the (co)homology of groups and associative algebras. Homol. Homot. Appl. 7(1), 87-108 (2005)

51. (With D. Arlettaz) Finite $K$-theory spaces. Math. Proc. Cambridge Philos. Soc. 139(2), 261-286(2005)

52. Equivariant homology and cohomology of groups. Topol. Appl. 153(1), 66-89 (2005)

53. (With T. Kandélaki) La conjecture de Karoubi pour la $K$-théorie lisse. C. R. Math. Acad. Sci. Paris 346(21-22), 1129-1132 (2008)

54. (With E. Khmaladze) Hopf formulas for equivariant integral homology of groups. Proc. Amer. Math. Soc. 138(9), 3037-3046 (2010)

55. (With T. Kandelaki and R. Meyer) Localisation and colocalisation of $K K$-theory. Abh. Math. Semin. Univ. Hambg. 81(1), 19-34 (2011)

56. (With T. Kandelaki) Smooth $K$-theory of locally convex algebras. Commun. Contemp. Math. 13(4), 553-577 (2011)

57. (With T. Kandelaki and R. Meyer) Localisation and colocalisation of triangulated categories and equivariant $K K$-theory. Proc. A. Razmadze Math. Inst. 155, 119-124 (2011)

58. (With T. Kandelaki and R. Meyer) Localisation and colocalisation of triangulated categories at thick subcategories. Math. Scand. 110(1), 59-74 (2012) 